\title{
GEMALS: A promising therapy for amyotrophic lateral sclerosis
}

\author{
MICHEL GEFFARD $^{1}$, ARTURO MANGAS ${ }^{1-3}$, DENIS BEDAT ${ }^{1}$ and RAFAEL COVEÑAS ${ }^{3}$ \\ ${ }^{1}$ Research Department, Institute for Development of Research on Human Pathology and Therapeutics, 33400 Talence; \\ ${ }^{2}$ Gemacbio, Research Department, Lieu dit Berganton, 33127 Saint Jean d'Illac, France; ${ }^{3}$ Institute of Neurosciences of \\ Castilla y León, Laboratory of Neuroanatomy of The Peptidergic Systems, University of Salamanca, 37007 Salamanca, Spain
}

Received February 15, 2017; Accepted September 14, 2017

DOI: $10.3892 / \mathrm{etm} .2018 .5868$

\begin{abstract}
Amyotrophic lateral sclerosis (ALS) is a neurodegenerative disease that currently has no cure. At present, the only approved treatment for ALS is Riluzole, a glutamate release blocker that improves life expectancy by 3-6 months. ALS-Endotherapia (GEMALS) is a novel therapeutic approach to treat ALS and the aim of the present study was to investigate the potential beneficial effects of this novel treatment. A total of 31 patients with ALS were assessed in the current study. Deceleration of the disease was observed in $83.87 \%(\mathrm{P}<0.0001)$ of patients and mean life expectancy was increased by 38 months. Motor functions, including breathing, walking, salivation, speech, swallowing and writing, were also improved in patients treated with GEMALS. The results of the present study demonstrate that long-term treatment with GEMALS has a curative effect in patients with ALS. Furthermore, the overall effectiveness of GEMALS was assessed using the ALS Assessment Questionnaire. The score improvement was 76.2 and $100 \%$ for men and women, respectively $(\mathrm{P}<0.0001)$, compared with the worldwide reference score. The present study provides a promising basis for the use of GEMALS as a therapeutic treatment for patients with ALS; however, these results must be confirmed in a double-blinded and randomized clinical trial.
\end{abstract}

\section{Introduction}

Amyotrophic lateral sclerosis (ALS) is a rapidly progressive neurodegenerative disease. Patients gradually lose the ability to control their muscles and 3-5 years following diagnosis, they typically experience respiratory failure and succumb (1). Patient's ALS Assessment Questionnaire (ALSAQ-40) scores decrease from 40 (normal motor capabilities) at the onset of

Correspondence to: Dr Michel Geffard, Research Department, Institute for Development of Research on Human Pathology and Therapeutics, 200 Avenue de Thouars, 33400 Talence, France E-mail:mg.idrpht@orange.fr

Key words: amyotrophic lateral sclerosis, endotherapia, poly-L-lysine, human disease to 0 (total loss of motor capabilities) over an average of 4.4 years $(1,2)$. At present, no treatments are able to prevent ALS progression. Riluzole is the only drug that is currently approved for the treatment of ALS and typically increases life expectancy by 3-6 months (3-7). Vitamin $\mathrm{E}$ is also often prescribed to patients with ALS due to its anti-oxidizing activity (8). Potential novel treatments for ALS, including gabapentine, copaxone, topiramate, creatine and the use of progenitor cells, have been assessed; however, no beneficial effects have been observed (9). Therefore, novel, effective treatments for ALS are required.

Endotherapia is a novel therapeutic strategy that has been used to treat chronic conditions, including autoimmune, neurodegenerative and proliferative diseases (10-12). This therapy uses small molecules, including vitamins, amino acids and fatty acids, linked to poly-L-lysine (PLL) (10-12). PLL linkage increases the availability of vitamins, amino acids and fatty acids in vivo (10-12). Two drug candidates, multiple sclerosis (MS)-Endotherapia (GEMSP) and ALS-Endotherapia (GEMALS), have been assessed in patients with MS and ALS, respectively (13-16). The beneficial effects of GEMALS on ALS have previously been demonstrated in a superoxide dismutase 1 (SOD1) rat model of ALS (12) and in a small clinical trial $(n=12)(11)$. In the rat model, GEMALS delayed the onset of disease and increased the rat's lifespan (12). In humans the results obtained were promising; $83 \%$ of patients exhibited stabilization or improvement to their functional capacities (11). Furthermore, treatment with GEMALS reversed the functional capacity impairment $(50.07 \%)$ and increased patient life expectancy by 52.57 months (11).

The aim of the present study was to confirm the beneficial action of GEMALS in a larger sample of patients with ALS $(n=31)$ and to further investigate the effect of GEMALS on specific motor parameters that were not assessed in the previous study (11).

\section{Patients and methods}

Patients. A total of 31 patients (10 women, 35.5\%; 21 men, $64.5 \%$ ) with ALS in the evolutive phase were enrolled in the present study (age range, 38-82 years). The patients were recruited from January 1994 to December 2011 (Table I). There were no exclusion criteria. ALS diagnosis was performed by a neurologist at the French University 
Table I. Data for patients with amyotrophic lateral sclerosis in the present study.

\begin{tabular}{|c|c|c|c|c|c|c|c|}
\hline \multirow[b]{2}{*}{ Patient (sex) } & \multicolumn{2}{|c|}{ Diagnosis } & \multicolumn{2}{|c|}{ GEMALS start } & \multicolumn{2}{|c|}{ GEMALS end } & \multirow{2}{*}{$\begin{array}{c}\text { Duration of } \\
\text { treatment (months) }\end{array}$} \\
\hline & Date & Age (years) & Score & Age (years) & Score & Age (years) & \\
\hline $1(\mathrm{M})$ & $11 / 09$ & 62 & 35.5 & 62 & 15 & 66 & 36 \\
\hline $2(\mathrm{M})$ & 01/08 & 60 & 31 & 63 & 13 & 65 & 28 \\
\hline $3(\mathrm{M})$ & $03 / 10$ & 53 & 37 & 53 & 14.5 & 56 & 32 \\
\hline $4(\mathrm{~F})$ & - & - & 17 & 64 & 15 & 64 & 7 \\
\hline $5(\mathrm{~F})$ & $05 / 10$ & 72 & 33 & 73 & 16 & 75 & 27 \\
\hline $6(\mathrm{~F})$ & $01 / 09$ & 59 & 12 & 60 & 12 & 62 & 19 \\
\hline $7(\mathrm{M})$ & 2007 & 65 & 27 & 69 & 23 & 71 & 23 \\
\hline $8(\mathrm{~F})$ & $10 / 08$ & 68 & 16 & 71 & 6 & 73 & 19 \\
\hline $9(\mathrm{M})$ & $09 / 10$ & 36 & 28 & 38 & 20 & 39 & 9 \\
\hline $10(\mathrm{M})$ & $01 / 94$ & 47 & 12 & 62 & 11 & 66 & 48 \\
\hline $11(\mathrm{M})$ & $01 / 04$ & 53 & 33 & 60 & 35.5 & 63 & 36 \\
\hline $12(\mathrm{M})$ & $08 / 03$ & 40 & 40 & 40 & 6.5 & 49 & 110 \\
\hline $13(\mathrm{M})$ & $01 / 94$ & 47 & 12 & 62 & 11 & 66 & 48 \\
\hline $14(\mathrm{M})$ & 01/01 & 57 & 40 & 57 & 6 & 69 & 140 \\
\hline $15(\mathrm{M})$ & - & - & 35 & 74 & 27 & 76 & 15 \\
\hline $16(\mathrm{~F})$ & - & - & 27.5 & 80 & 24 & 82 & 15 \\
\hline $17(\mathrm{M})$ & 03/08 & 44 & 31 & 46 & 17 & 49 & 24 \\
\hline $18(\mathrm{~F})$ & 01/09 & 55 & 34 & 57 & 22 & 59 & 19 \\
\hline $19(\mathrm{~F})$ & - & - & 37 & 63 & 32 & 64 & 11 \\
\hline $20(\mathrm{M})$ & 06/07 & 65 & 33 & 69 & 25 & 71 & 32 \\
\hline $21(\mathrm{M})$ & 07/07 & 61 & 34 & 65 & 18 & 67 & 26 \\
\hline $22(\mathrm{~F})$ & - & - & 38 & 59 & 34.5 & 60 & 13 \\
\hline $23(\mathrm{M})$ & $02 / 08$ & 73 & 37 & 74 & 25 & 76 & 21 \\
\hline $24(\mathrm{M})$ & $08 / 05$ & 58 & 32 & 61 & 28 & 63 & 25 \\
\hline $25(\mathrm{~F})$ & $11 / 08$ & 44 & 35 & 47 & 36 & 49 & 28 \\
\hline $26(\mathrm{M})$ & $04 / 10$ & 62 & 26 & 64 & 12 & 64 & 9 \\
\hline $27(\mathrm{M})$ & $12 / 11$ & 68 & 27 & 70 & 23 & 70 & 3 \\
\hline $28(\mathrm{M})$ & $03 / 11$ & 58 & 34 & 59 & 34 & 60 & 8 \\
\hline $29(\mathrm{M})$ & $07 / 07$ & 57 & 29 & 59 & 17 & 63 & 50 \\
\hline $30(\mathrm{~F})$ & 03/07 & 32 & 39 & 33 & 0 & 38 & 66 \\
\hline $31(\mathrm{M})$ & 01/01 & 46 & 20 & 53 & 28 & 58 & 57 \\
\hline
\end{tabular}

M, male; F, female; GEMALS, amyotrophic lateral sclerosis-Endotherapia.

hospitals located in Nantes, Marseille, Nîmes, Versailles, Dijon, Henry-Mondor de Créteil, Nice, Grenoble, Paris, Lille, Poitiers, Toulouse, St Etienne, Rennes, Metz, Montpellier and Angers using standardised clinical criteria and electromyogram tests as previously described (17). The study was national, multicenter, non-randomized and non-blinded. The duration of GEMALS treatment was between 3 and 140 months (Table I) and patients were evaluated 3 or 4 times per year. The following data were collected for each patient: Age, sex and date of diagnosis, start/finish of GEMALS treatment and ALS Assessment Questionnaire (ALSAQ-40) scores over time (Table I). All patients provided prior informed written consent for inclusion in the current study.

GEMALS synthesis and dose. A dose of (60-180 $\mu \mathrm{g} / \mathrm{kg} / \mathrm{day})$ GEMALS was administered via the sublingual route. Initially all patients were administered $60 \mu \mathrm{g} / \mathrm{kg} / \mathrm{day}$, however the dosage was doubled (when there was a loss of 1 point of the ALSQ-40 score in 1 month) or tripled (when doubled dose was not enough to reduce the deterioration and there was a decrease of $\geq 1$ point of the ALSQ-40 score in 1 month) if the patients condition deteriorated. As previously described, GEMALS was synthesized according to patent numbers 792167 (EU) and 6114388 (USA) (12) and purchased from Farmacia Legnani (Milan, Italy). GEMALS contains a mixture of functional polypeptides: Fatty acids (including thioctic acid and oleic acid), antioxidants (including ascorbic acid), free radical scavengers and amino acids (including taurine and methionine), all linked to PLL (Table II). If patients presented with an acceleration of the disease during follow up, the dosage of GEMALS was doubled to maintain its protective effect. 
Table II. Amyotrophic lateral sclerosis-Endotherapia constituents.

Final

concentration

PLL constituents

(M)

\begin{tabular}{ll}
\hline Glucosamine-PLL & $1 \times 10^{-04}$ \\
Agmatine-PLL & $1 \times 10^{-04}$ \\
Uric acid-PLL & $1 \times 10^{-04}$ \\
$\alpha$-Tocopherol-PLL & $3 \times 10^{-05}$ \\
Ascorbic acid-PLL & $3 \times 10^{-05}$ \\
CoEnzyme Q10-PLL-oleic acid & $3 \times 10^{-05}$ \\
Retinoic acid-PLL-oleic acid & $3 \times 10^{-05}$ \\
Pantothenic acid-PLL & $3 \times 10^{-05}$ \\
Biotin-PLL & $3 \times 10^{-05}$ \\
Oleic acid-PLL-thioctic acid & $1 \times 10^{-04}$ \\
Oleic acid-PLL-myristic acid & $1 \times 10^{-04}$ \\
Oleic acid-PLL-lauric acid & $1 \times 10^{-04}$ \\
Oleic acid-PLL-linoleic acid & $1 \times 10^{-04}$ \\
Oleic acid-PLL-palmitic acid & $1 \times 10^{-04}$ \\
Oleic acid-PLL-palmitoleic acid & $1 \times 10^{-04}$ \\
Lauric acid-PLL-caprylic acid & $1 \times 10^{-04}$ \\
T-T-Farnesyl-L. cysteine-PLL-palmitic acid & $1 \times 10^{-04}$ \\
Cholesterol-PLL-oleic acid & $1 \times 10^{-04}$ \\
L. Cysteine-PLL & $2 \times 10^{-04}$ \\
Taurine-PLL & $2 \times 10^{-04}$ \\
L. Methionine-PLL & $2 \times 10^{-04}$ \\
L. Glutathione-PLL & $1 \times 10^{-04}$ \\
\hline
\end{tabular}

PLL, poly-L-lysine.

Score. ALS evaluation was based on the evolution of ALSAQ-40 scores throughout the follow-up period, with scores ranging from 40 (normal) to 0 (total loss of motor capabilities). Two ALSAQ-40 scores were defined and compared: M score, which was the score of each patient in the present study determined during follow-up visits; R score, which was the worldwide reference score of theoretical disease progression without treatment (mean ALSAQ-40 score decrease is 0.769 points/month). Each score was measured as previously described $(18,19)$. ALSAQ-40 is a patient health status report used to measure the subjective well being of ALS patients and is primarily used in clinical trials for novel ALS drugs (20,21). ALSAQ-40 is sensitive, reliable and an acceptable tool for the assessment of quality of life in patients with ALS $(2,20)$. To estimate the effect of GEMALS treatment, a reference curve of the mean individual evolution of ALSAQ-40 score over time was constructed. Patient responses were evaluated as follows: Progression, the individual mean speed of evolution of ALSAQ-40 score with GEMALS was $\leq \mathrm{R}$ score (-0.769 points/month); decrease, the individual mean speed of evolution of ALSAQ-40 score with GEMALS was between -0.769 and 0 points/month; stabilization, the individual mean speed of evolution of ALSAQ-40 score with GEMALS was 0 points/month; and improvement, the individual mean speed of evolution of ALSAQ-40 score with GEMALS was $>0$ points/month.
Statistical analysis. Due to the high variability of the ALS cohort included in the present study, a previously published method of adapted valuation was used (11). The Mann-Whitney $\mathrm{U}$ test was used to compare the study population with the reference population. Analyses were performed using SAS Version 9 (SAS Institute, Inc., Cary, NC, USA) and statistical analysis was conducted externally by Stalphamis (Le Bourg, France). $\mathrm{P}<0.05$ was considered to indicate a statistically significant difference.

A comprehensive study of the ALSAQ-40 score, ALSAQ-40 evolution and the evolution of the ALSAQ-40 score based on each item was performed. For descriptive analysis, the following parameters were investigated: Strength, percentage, distribution, minimum, 1st quartile, median, 3rd quartile, maximum, mean, standard deviation and $95 \%$ confidence intervals. For comparative analysis, Student's t test was used to compare final $\mathrm{M}$ and $\mathrm{R}$ scores. The equality of variance was verified using the folded $\mathrm{F}$ test. Evolution of the ALSAQ-40 score was analyzed using a linear regression model and the following parameters were used to analyze $M$ and R scores: Coefficient of correlation $\left(\mathrm{R}^{2}\right)$ and associated $\mathrm{P}$-value, intercept and slope $(\mathrm{ax}+\mathrm{b})$. A $\chi^{2}$ or Fisher's exact test were used to compare success and failure rates according to the theoretical strength obtained by unilateral assumption (\% success $>\%$ of failure).

\section{Results}

General considerations. In the 31 patients treated with GEMALS, $16.13 \%$ experienced a worsening of their condition (degradation), $70.97 \%$ experienced a decrease in the progression of the disease (deceleration) and $12.90 \%$ experienced a reversal of disease evolution (improvement) (Fig. 1 and Table III). When the final score was higher than the initial score, the disease was considered to have improved. When the initial and final scores were equal or the final $\mathrm{M}$ score was higher than the $\mathrm{R}$ score, the disease was considered to have decelerated. When the final $\mathrm{M}$ score was lower than the $\mathrm{R}$ score, the evolution was considered have degraded (Fig. 1 and Table III). The mean duration of GEMALS treatment was of 988 days with a standard deviation of 902 days (minimum, 98 days; maximum, 4,287 days). The mean score evolution of patients treated with GEMALS was -0.40 points/month and the worldwide reference score evolution was -0.77 points/month (Fig. 2); this represents a significant increase in life expectancy of 38 months (3.16 years). A total of $83.87 \%$ of patients treated with GEMALS had an improvement in the evolution of ALS following GEMALS treatment $(\mathrm{P}<0.0001$; Table III). Additionally, no side effects were reported and following rigorous questioning of the patient no clinical symptoms including, cutaneous alterations, flu-like symptoms, gastrointestinal troubles, migraines, cephalea, insomnia, nauseas and vomiting were described. Additionally the biological parameters (blood cell count, ionograms, lipidic balance, hepatic enzymes, renal filtration or glycaemia) were unaltered.

Item scores. Following GEMALS treatment, the M score was significantly higher than the R score for the following functions: Speech $(\mathrm{P}<0.005)$, salivation $(\mathrm{P}<0.001)$, swallowing $(\mathrm{P}<0.001)$, writing $(\mathrm{P}<0.05)$, ability to turn in bed $(\mathrm{P}<0.05)$, 
Table III. Effects of amyotrophic lateral sclerosis-Endotherapia treatment $(n=31)$.

\begin{tabular}{lccrr}
\hline & \multicolumn{3}{c}{ No. patients $(\%)$} & Success $(\%)$ \\
\cline { 2 - 4 } Evolution & Male $(\mathrm{n}=21)$ & Female $(\mathrm{n}=10)$ & Total & P-value \\
\hline Improvement & $2(9.52)$ & $2(20.00)$ & $4(12.90)$ & $26 / 31(83.87)$ \\
Deceleration & $14(66.67)$ & $8(80.00)$ & $22(70.97)$ & $<0.0001$ \\
Degradation & $5(23.80)$ & $0(0)$ & $5(16.13)$ & \\
\hline
\end{tabular}

Success rate $=($ improvement + deceleration $) /$ total. The P-value presented in this table is for success rate vs. degradation.
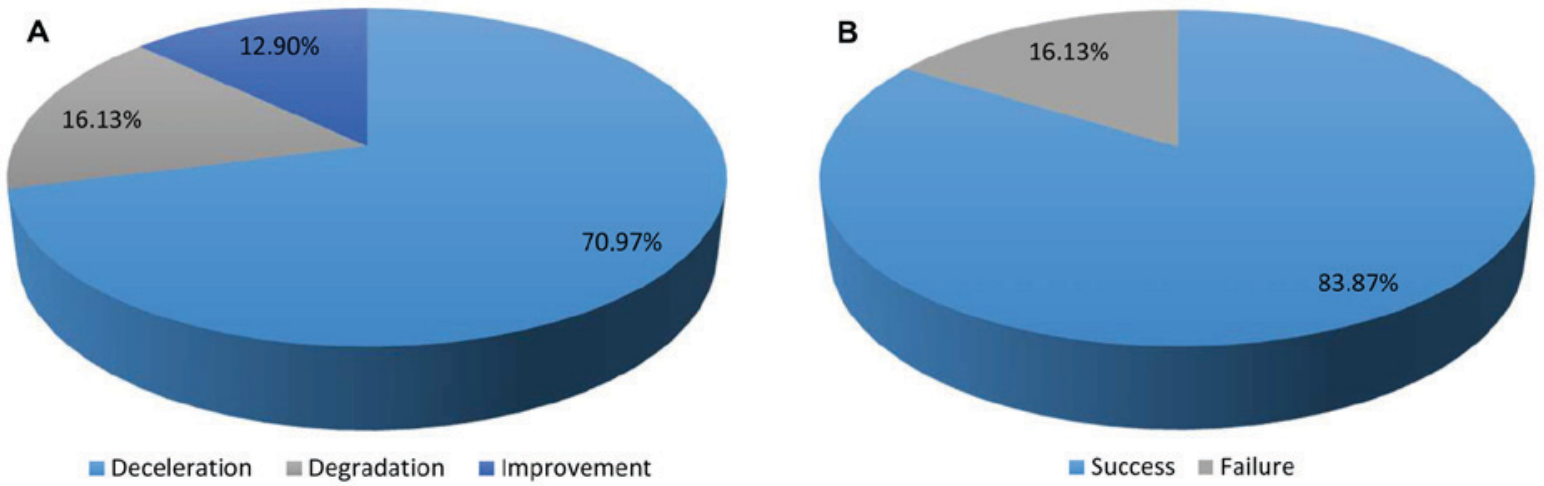

Figure 1. (A) Proportion of patients that experienced deceleration, degradation and improvement. (B) Success and failure rates of treatment. $n=31$.

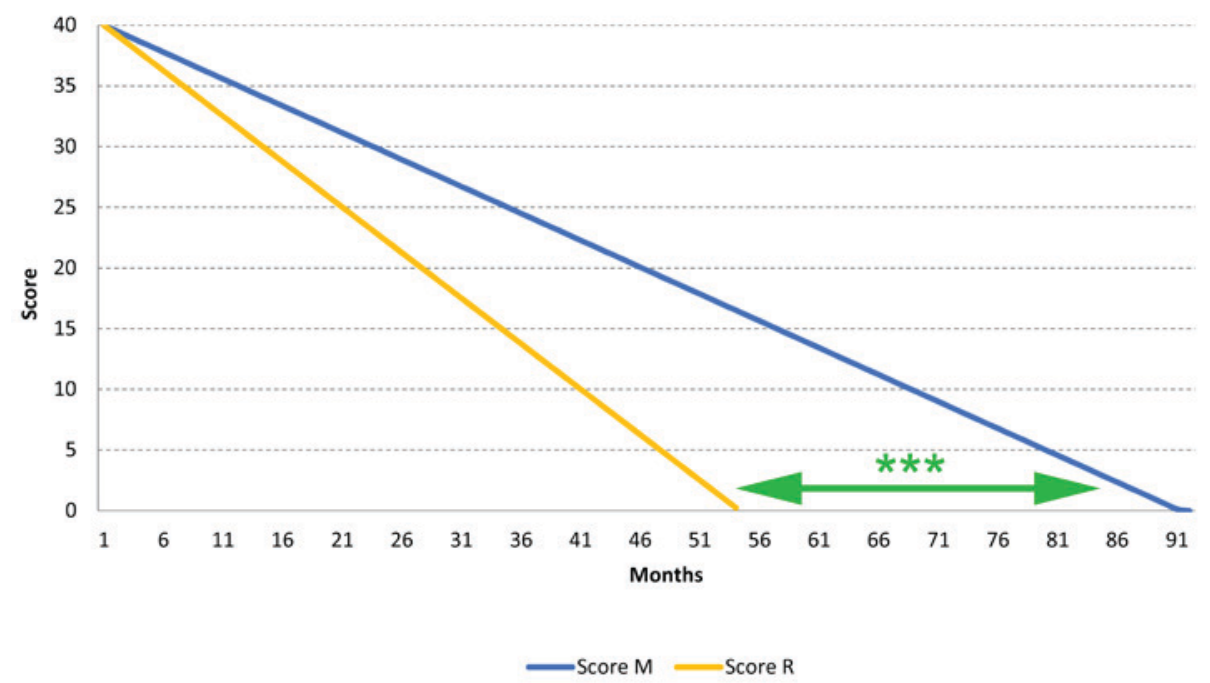

Figure 2. Mean change in amyotrophic lateral sclerosis Assessment Questionnaire score. M, patients treated with amyotrophic lateral sclerosis-endotherapia in the present study; R, worldwide reference score. An increase in life expectancy of 38 months was observed in patients treated with GEMALS (score M) in comparison with worldwide reference score (score R). ${ }^{* * *} \mathrm{P}=0.0002$.

walking $(\mathrm{P}<0.01)$ and breathing $(\mathrm{P}<0.001)($ Table IV). No significant differences between $\mathrm{M}$ and $\mathrm{R}$ scores were observed for ability to cut food, ability to dress and ability to climb stairs (Table IV).

Item score evolutions. Patients were considered to have improved if there was an increase in the $\mathrm{M}$ score over time. If the M score decreased over time and this decrease was
$<0.769$ points/month, patients were considered to have decelerated. Patients were considered to have degraded if the $\mathrm{M}$ score decreased throughout the treatment period at $>0.769$ points/month. Improvement and deceleration indicate treatment success and degradation indicates treatment failure. Items with a success rate $>50 \%$ are presented in Fig. 3. The results indicate that the success rate for breathing was significantly greater than the failure rate $(\mathrm{P}<0.05$; Fig. 3$)$. 
Table IV. Scores according to each item.

\begin{tabular}{lccc}
\hline Item & Inclusion & $\mathrm{M}$ & $\mathrm{R}$ \\
\hline Speech & $2.9 \pm 1.4$ & $2.3 \pm 1.7^{\mathrm{c}}$ & $1.2 \pm 1.1$ \\
Salivation & $3.3 \pm 0.9$ & $2.6 \pm 1.4^{\mathrm{d}}$ & $1.3 \pm 1.1$ \\
Swallowing & $3.4 \pm 0.9$ & $2.8 \pm 1.3^{\mathrm{d}}$ & $1.4 \pm 1.1$ \\
Writing & $2.9 \pm 1.0$ & $1.8 \pm 1.6^{\mathrm{a}}$ & $1.1 \pm 1.1$ \\
Ability to cut food & $2.6 \pm 1.3$ & $1.5 \pm 1.3$ & $0.9 \pm 1.0$ \\
Ability to dress & $2.6 \pm 1.2$ & $1.3 \pm 1.2$ & $1.0 \pm 1.1$ \\
Ability to turn in bed & $3.0 \pm 1.3$ & $2.0 \pm 1.5^{\mathrm{a}}$ & $1.2 \pm 1.2$ \\
Walking & $2.6 \pm 0.9$ & $1.7 \pm 1.1^{\mathrm{b}}$ & $1.0 \pm 1.0$ \\
Ability to climb stairs & $2.2 \pm 1.3$ & $1.1 \pm 1.2$ & $0.9 \pm 1.0$ \\
Breathing & $3.1 \pm 1.2$ & $2.7 \pm 1.4^{\mathrm{d}}$ & $1.3 \pm 1.2$ \\
\hline
\end{tabular}

Data are presented as the mean \pm standard deviation. ${ }^{\mathrm{a}} \mathrm{P}<0.05$, ${ }^{\mathrm{b}} \mathrm{P}<0.01,{ }^{\mathrm{c}} \mathrm{P}<0.005$ and ${ }^{\mathrm{d}} \mathrm{P}<0.001 \mathrm{vs}$. $\mathrm{R}$. The inclusion values indicate the mean $\mathrm{M}$ score \pm standard deviation of patients at the beginning of the study.

Overall study. Overall evolutions for each patient were calculated by linear regression, the evolution category (improvement, deceleration and degradation) mean values are presented in Table V. The mean overall M score was 19.8 , which was significantly higher than the mean overall R score of 11.7 $(\mathrm{P}=0.0013)$. The mean values at the end of the study represent an amelioration of the $\mathrm{M}$ group vs. the $\mathrm{R}$ group at $30.8 \%$ $(\mathrm{P}=0.0002)$. The study population included 10 women and 21 men; the success rate of treatment was $100 \%$ for women and $76.19 \%$ for men (Table III; $\mathrm{P}<0.0001$ ). Overall the treatment was significantly successful ( $\mathrm{P}<0.0001$; Table III and Fig. 4).

\section{Discussion}

In a previous study using a SOD1 rat model, it was demonstrated that GEMALS significantly extended lifespans and improved electromyographic parameters (12). However, no significant increase in life expectancy was observed when rats were treated with Riluzole or other pharmacological agents, including minocycline and nifedipine (12). Two GEMALS concentrations were also assessed and the results suggested that the effects of GEMALS may be dose-dependent (12). Based on these results, the higher dose $(3.75 \mathrm{mg} / \mathrm{ml})$ administered to SOD1 rats was extrapolated for use in the present study in humans (4-12.05 mg/day). In the SOD1 animal model and humans GEMALS demonstrated a beneficial effect.

The effect of GEMALS in 12 patients with ALS has been previously reported (11). The results indicated that GEMALS treatment caused a $50.07 \%$ decrease in the loss of functional capacities, corresponding to a mean life expectancy increase of 52.57 months (4.38 years). In the present study, there was an increase in life expectancy of 38 months (3.16 years). Each study indicates that GEMALS is an effective method of treating ALS, with beneficial effects observed in $83 \%$ of patients in the previous report (11) and $83.7 \%$ in the present study. In the previous study (11), the ALSAQ40 was reported; however, data regarding specific functions, including speech, were not available. In the present study, the effect of GEMALS treatment on specific functions was evaluated and significant improvements in breathing, walking, salivation, speech, swallowing and writing were observed.

The glutamate release blocker Riluzole is, at present, the only authorized treatment for ALS; however, Riluzole treatment extends life expectancy by only a few months (3). It has been demonstrated that treatment with Riluzole for 12 months reduces deterioration of muscular function by $33.4 \%$ and extends life expectancy by 2.77 months (4). It has also been reported that treatment with Riluzole for 12 months extends survival by 4.2 months; however, this advantageous effect is transient and disappears when the monitoring period is extended (5). This transient effect has been reported in another study, with Riluzole only effective at treating patients affected by the bulbar form of ALS, which represents $30 \%$ of all ALS cases (18).

It has previously been reported that GEMALS treatment decreases the loss of functional capacities that occurs in patients with ALS (11). No side effects have been reported following treatment with GEMALS or GEMSP (13). Similarly, in the current study, no side effects following GEMALS treatment were reported. This may be due to the low dose of GEMALS received by each patient. The individual doses administered were calculated according to the evolution of each patient at follow-up; thus, doses ranged from 60 to $180 \mu \mathrm{g} / \mathrm{kg} /$ day. PLL accounts for $\sim 90 \%$ of the drug molecular weight, therefore only $0.4-1.2 \mathrm{mg}$ of small molecules were delivered per administration.

To overcome the high variability of the ALS cohort studied here, the Mann-Whitney U test was used as previously reported (11). A total of 21 men and 10 women were included in the present study and the treatment success rates were 76.2 and $100 \%$, respectively, however future studies should use a larger population size to confirm the results of the present study. Compared with Riluzole therapy, GEMALS increased the life expectancy of patients with ALS from 4 to 38 months (3). Compared with the GEMALS pilot study (11), the life expectancy of patients decreased from 52.57 to 38 months. However, if patients 26 and 27, who did not respond to treatment with GEMALS, were excluded from further analyses, the mean increase in life expectancy in the current study was 89 months $(n=29)$.

The activity of Riluzole is based on the blockage of excitatory amino acid pathways. It blocks sodium dependent voltage channels, thus decreasing the release of glutamate (22). Taurine, one of the constituents of GEMALS, also reduces glutamate excitotoxicity by enhancing mitochondrial function and regulating intracellular calcium homeostasis (23). ALS is a multifactorial disease in which oxidative stress, mitochondrial damage, neuroinflammation, zinc excitotoxicity and apoptosis serve important roles. Treatment of ALS therefore requires a multifactorial strategy; GEMALS, a tailor-made combination therapy composed of functional polypeptides, was developed for this purpose. In the present study, the constituents of GEMALS were rigorously selected according to their known effects on the regeneration or protection of neuronal components $(10,11)$. These constituents were selected with the aim of decreasing oxidative and free radical stress, reducing inflammation, inhibiting exogenous toxic factors and exhibiting neuroprotective effects (12). 
Table V. Global evolution mean values.

Mean values

\begin{tabular}{|c|c|c|c|c|c|c|c|}
\hline Evolution & No. cases & $\mathrm{R}_{2} \mathrm{M}$ Group & P-value & M Slope & $\mathrm{R}_{2} \mathrm{R}$ Group & P-value & R Slope \\
\hline Improvement & 4 & 0.58 & 0.125 & 0.00234 & -0.98 & $<0.0004$ & -0.02075 \\
\hline Deceleration & 22 & -0.86 & $<0.049$ & -0.01357 & -0.98 & $<0.0003$ & -0.02325 \\
\hline Degradation & 5 & -0.93 & $<0.040$ & -0.03118 & -0.94 & $<0.0001$ & -0.02120 \\
\hline
\end{tabular}

A
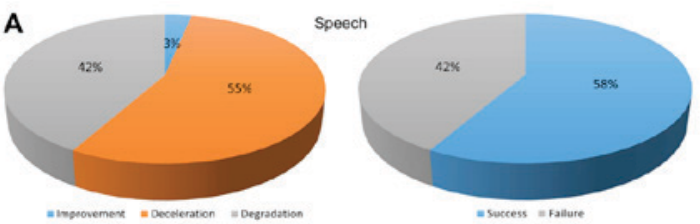

C

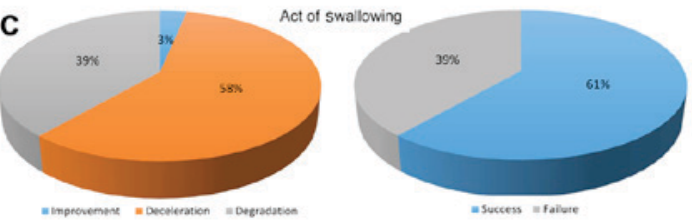

E

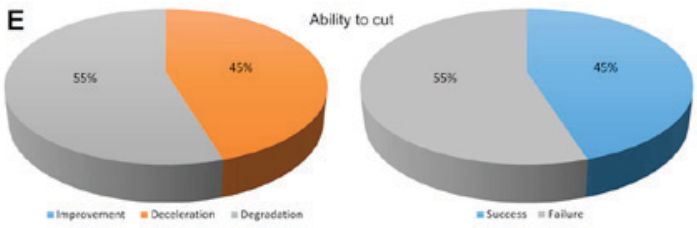

G
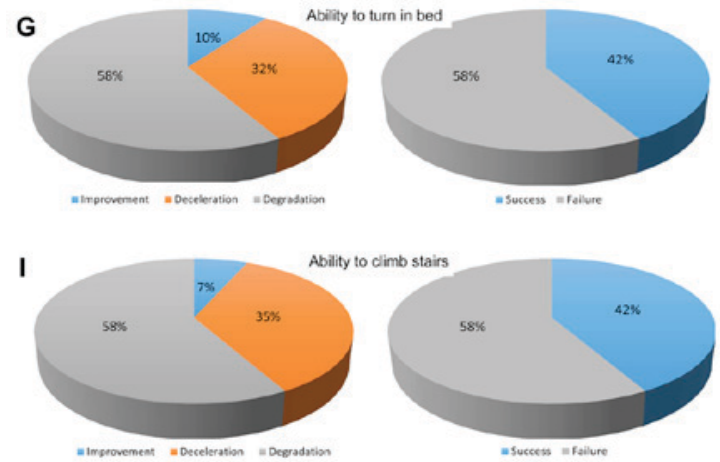

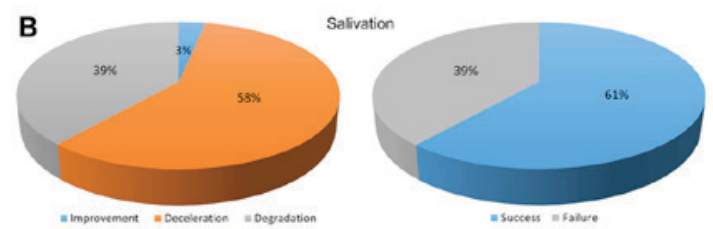

D

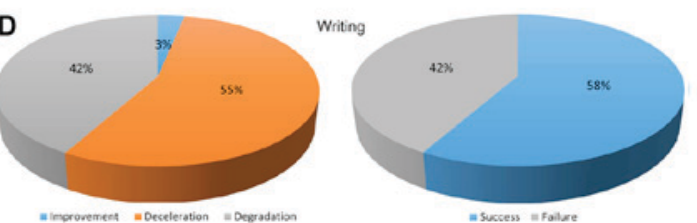

$\mathbf{F}$

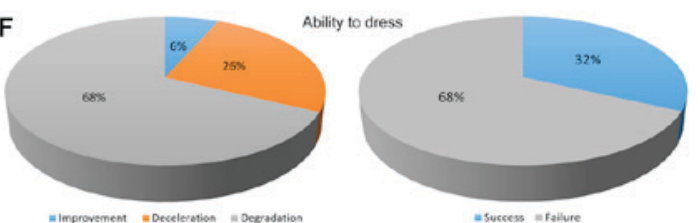

H

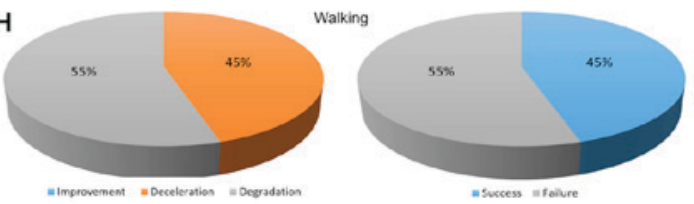

$J$

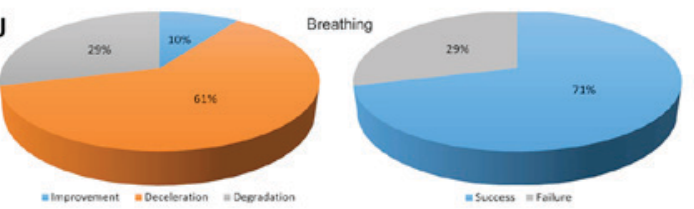

Figure 3. Evolution of important motor functions according to in amyotrophic lateral sclerosis Assessment Questionnaire score. (A) Speech, (B) salivation, (C) swallowing, (D) writing, (E) ability to cut food, (F) ability to dress, (G) ability to turn in bed, (H) walking, (I) ability to climb stairs and (J) breathing.

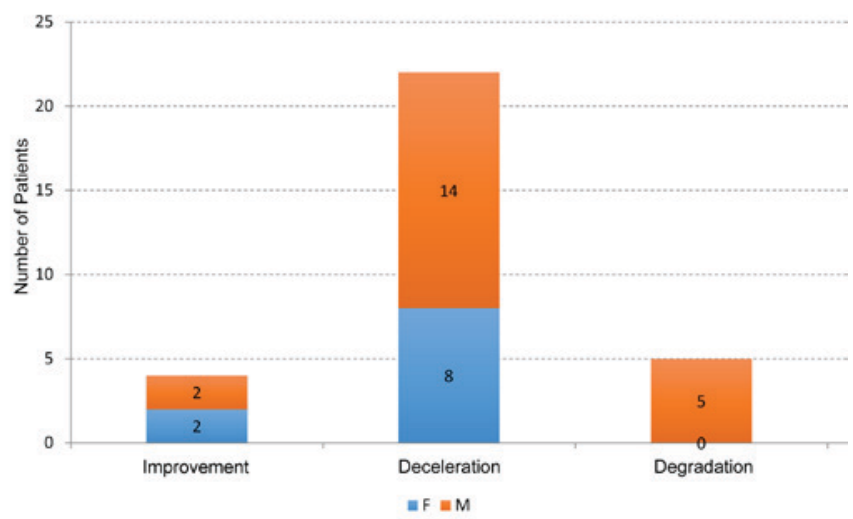

Figure 4. Disease evolution and sex. F, female; $M$, male. $n=31$.
All PLL-linked molecules used in the present study are endogenous and non-immunogenic, allowing for long-term administration without the induction of toxicity and/or side effects. The quantities of PLL-linked molecules represent a low proportion (w/w) of the overall dosage, which may account for the absence of side effects following treatment with GEMALS and GEMSP (11). Free components not linked to PLL have been reported to have no therapeutic effect in experimental animal models $(14-16,24)$. PLL therefore appears to be more than a simple vector; PLL compounds exhibit therapeutic properties. PLL-linked molecules are able to cross the blood brain barrier, which is important as certain molecules, including butyrilcholinesterase (molecular weight, $340 \mathrm{KDa}$ ), are unable to do so alone (25). Furthermore, PLL-linkage may increase the active 
duration of molecules; for example, PLL-linked butyrilcholinesterase remains active for $\geq 72 \mathrm{~h}$ (25). The results of a previous study investigating GEMSP indicated that methionine-PLL, a constituent of GEMSP, was present in the motor neurons of the spinal cord (15), exerting potential neuroprotective action. This observation is similar to that of a different study, in which PLL was identified inside organelles and the nuclear membrane (26).

The constituents of GEMALS exert the following actions: i) Poly-unsaturated fatty acids exert a neuroprotective effect and act as free radical scavengers to prevent oxidation of unsaturated fatty acids in the cell membrane $(27,28)$; ii) $\alpha$-tocopherol exerts a neuroprotective effect by potentially attenuating the entry of $\mathrm{Ca}^{2+}$ via transient receptor potential-like channels (29); iii) ascorbic acid is necessary for the maintenance of neuronal functions and exerts an antioxidant effect (30); iv) polyamine agmatine exerts a neuroprotective effect against the excitotoxic action of glutamate (31); v) cysteine, methionine, glutathione, retinoic acid, pantothenic acid and biotin act as antioxidants and scavengers to reduce the apoptosis and neuronal death induced by reactive oxygen species (32); and vi) taurine modulates mitochondrial calcium homeostasis (33).

In conclusion, a multifactorial strategy for the treatment of ALS is required and GEMALS may be an effective treatment. The present study is an in-depth investigation demonstrating and confirming the effectiveness of GEMALS in a larger cohort of patients with ALS. The results suggest that GEMALS treatment significantly increases life expectancy and decreases the loss of important motor functions in patients with ALS; however, a phase IIb multicentric, randomized and double-blinded clinical trial is required to confirm these results.

\section{Acknowledgements}

The present study was supported by the Institut pour le Développement de la Recherche en Pathologie Humaine et Thérapeutique. The authors would like to thank Mr. B. Combes (Stalphamis; Le Bourg, France) for conducting statistical analysis.

\section{References}

1. Shamshiri H, Fatehi F, Abolfazli R, Harirchian MH, Sedighi B, Zamani B, Roudbari A, Razazian N, Khamseh F and Nafissi S: Trends of quality of life changes in amyotrophic lateral sclerosis patients. J Neurol Sci 368: 35-40, 2016.

2. Jenkinson C, Norquist JM and Fitzpatrick R: Deriving summary indices of health status from the amyotrophic lateral sclerosis assessment questionnaires (ALSAQ-40 and ALSAQ-5). J Neurol Neurosurg Psychiatry 74: 242-245, 2003.

3. Boillée S and Lobsiger CS: Glial cells not that supportive for motor neurons. Med Sci (Paris) 24: 124-126, 2008 (In French).

4. Bensimon G, Lacomblez L and Meininger V: A controlled trial of Riluzole in amyotrophic lateral sclerosis. ALS/Riluzole Study Group. New J Eng Med 330: 585-591, 1994.

5. Traynor BJ, Alexander M, Corr B, Frost E and Hardiman O: An outcome study of riluzole in amyotrophic lateral sclerosis - a population-based study in Ireland, 1996-2000. J Neurol 250: 473-479, 2003.

6. Meininger V, Lacomblez L and Salachas F: What has changed with riluzole? J Neurol 247: 19-22, 2000.

7. Rossi S: Australian medicines handbook. Australian Med Handbook Unit Trust Adelaide, 2013.
8. Ascherio A, Weisskopf MG, O'reilly EJ, Jacobs EJ, McCullough ML, Calle EE, Cudkowicz M and Thun MJ: Vitamin E intake and risk of amyotrophic lateral sclerosis. Ann Neurol 57: 104-110, 2005.

9. Jones SP, Guillemin GJ and Brew BJ: The kynurenine pathway in stem cell biology. Int J Tryptophan Res 6: 57-66, 2013.

10. Geffard M, de Bisschop L, Duleu S, Hassaine N, Mangas A and Coveñas R: Endotherapia: A new frontier in the treatment of multiple sclerosis and other chronic diseases. Discov Med 10: 443-451, 2010.

11. Geffard M, de Bisschop L, Duleu S, Pouns O, Ferran G, Bessede A, Hassaine N, Autran JL, Bodet D, Mangas A and Covenas R: Endotherapia. Antiinflamm Antiallergy Agents Med Chem 9: 197-211, 2010.

12. Nicaise C, Coupier J, Dabadie MP, De Decker R, Mangas A, Bodet D, Poncelet L, Geffard M and Pochet R: Gemals, a new drug candidate, extends lifespan and improves electromyographic parameters in a rat model of amyotrophic lateral sclerosis. Amyotroph Lateral Scler 9: 85-90, 2008.

13. Geffard M, Duleu S, Bessede A, Vigier V, Bodet D, Mangas A and Coveñas R: GEMSP: A new therapeutic approach of multiple sclerosis. Cent Nerv Syst Agents Med Chem 12: 173-181, 2012.

14. Mangas A, Covenas R, Bodet D, Dabadie MP, Glaize G and Geffard M: Evaluation of the effects of a new drug on brain leukocyte infiltration in an experimental model of autoimmune encephalomyelitis. Lett Drug Des Discov 3: 138-148, 2006.

15. Mangas A, Coveñas R, Bodet D, de León M, Duleu S and Geffard M: Evaluation of the effects of a new drug candidate (GEMSP) in a chronic EAE model. Int J Biol Sci 4: 150-160, 2008.

16. Mangas A, Coveñas R, Bodet D, Duleu S and Geffard M: A new drug candidate (GEMSP) for multiple sclerosis. Curr Med Chem 16: 3203-3214, 2009.

17. Couratier P, Marin B, Laurette G, Nicol M and Preux PM: Epidemiologie, clinical spectrum of ALS and differential diagnoses. Presse Med 43: 538-548, 2014 (In French).

18. Kimura F, Fujimura C, Ishida S, Nakajima H, Furutama D, Uehara H, Shinoda K, Sugino M and Hanafusa T: Progression rate of ALSFRS-R at time of diagnosis predicts survival time in ALS. Neurology 66: 265-267, 2006.

19. Labra J, Menon P, Byth K, Morrison S and Vucic S: Rate of disease progression: A prognostic biomarker in ALS. J Neurol Neurosurg Psychiatry 87: 628-632, 2016.

20. Jenkinson C, Fitzpatrick R, Brennan C, Bromberg M and Swash M: Development and validation of a short measure of health status for individuals with amyotrophic lateral sclerosis/motor neurone disease: The ALSAQ-40. J Neurol 3 (246 Suppl): III16-III21, 1999.

21. Jenkinson C, Fitzpatrick R, Brennan C and Swash M: Evidence for the validity and reliability of the ALS assessment questionnaire: The ALSAQ-40. Amyotroph Lateral Scler Other Motor Neuron Disord 1: 33-40, 1999.

22. Dolfi SC, Medina DJ, Kareddula A, Paratala B, Rose A, Dhami J, Chen S, Ganesan S, Mackay G, Vazquez A and Hirshfield KM: Riluzole exerts distinct antitumor effects from a metabotropic glutamate receptor 1-specific inhibitor on breast cancer cells. Oncotarget 8: 44639-44653, 2017.

23. El Idrissi A: Taurine increases mitochondrial buffering of calcium: Role in neuroprotection. Amino Acids 34: 321-328, 2008.

24. Mangas A, Coveñas R and Geffard M: New drug therapies for multiple sclerosis. Curr Opin Neurol 23: 287-292, 2010.

25. Gaydess A, Duysen E, Li Y, Gilman V, Kabanov A, Lockridge O and Bronich T: Visualization of exogenous delivery of nanoformulated butyrylcholinesterase to the central nervous system. Chem Biol Interact 187: 295-298, 2010.

26. Wang Z, Zhu H, Li D and Yang X: Preparation and application of single polyelectrolyte microcapsules possessing tunable autofluorescent properties. Colloids Surfaces A: Physicochem Eng Aspects 329: 58-66, 2008.

27. Blondeau N, Widmann C, Lazdunski M and Heurteaux C: Polyunsaturated fatty acids induce ischemic and epileptic tolerance. Neuroscience 109: 231-241, 2002.

28. Yoshida H, Yanai H, Namiki Y, Fukatsu-Sasaki K, Furutani N and Tada N: Neuroprotective effects of edaravone: A novel free radical scavenger in cerebrovascular injury. CNS Drug Rev 12: 9-20, 2006. 
29. Crouzin N, de Jesus Ferreira MC, Cohen-Solal C, Aimar RF Vignes $\mathrm{M}$ and Guiramand J: Alpha-tocopherol-mediated long-lasting protection against oxidative damage involves an attenuation of calcium entry through TRP-like channels in cultured hippocampal neurons. Free Radic Biol Med 42: 1326-1337, 2007.

30. Qiu S, Li L, Weeber EJ and May JM: Ascorbate transport by primary cultured neurons and its role in neuronal function and protection against excitotoxicity. J Neurosci Res 85: 1046-1056, 2007.

31. Wang WP, Iyo AH, Miguel-Hidalgo J, Regunathan S and Zhu MY: Agmatine protects against cell damage induced by NMDA and glutamate in cultured hippocampal neurons. Brain Res 1084: 210-216, 2006.
32. Agar $\mathbf{J}$ and Durham $\mathrm{H}$ : Relevance of oxidative injury in the pathogenesis of motor neuron diseases. Amyotroph Lateral Scler Other Motor Neuron Disord 4: 232-242, 2003.

33. Kumari N, Prentice $\mathrm{H}$ and Wu JY: Taurine and its neuroprotective role. Adv Exp Med Biol 775: 19-27, 2013.

cc) (i) (9) This work is licensed under a Creative Commons Attribution-NonCommercial-NoDerivatives 4.0 International (CC BY-NC-ND 4.0) License. 